\title{
Germanica
}

\section{Gattungsdenken im Umbruch. Zum Lyrik-Diskurs der deutschen Literaturwissenschaft nach 1945}

Repenser la notion de genre. Sur le discours lyrique des sciences littéraires après 1945

\section{Ludwig Volker}

\section{OpenEdition}

\section{Journals}

Édition électronique

URL : http://journals.openedition.org/germanica/1488

DOI : 10.4000/germanica. 1488

ISSN : 2107-0784

\section{Éditeur}

Université de Lille

\section{Édition imprimée}

Date de publication : 30 décembre 1997

Pagination : 163-177

ISBN : 977098426320321

ISSN : 0984-2632

Référence électronique

Ludwig Volker, « Gattungsdenken im Umbruch. Zum Lyrik-Diskurs der deutschen

Literaturwissenschaft nach 1945 », Germanica [Online], 21 | 1997, Online erschienen am: 20 November 2015, abgerufen am 06 Oktober 2020. URL : http://journals.openedition.org/germanica/1488 ; DOI https://doi.org/10.4000/germanica.1488

Ce document a été généré automatiquement le 6 octobre 2020.

(c) Tous droits réservés 


\title{
Gattungsdenken im Umbruch. Zum Lyrik-Diskurs der deutschen Literaturwissenschaft nach 1945
}

\author{
Repenser la notion de genre. Sur le discours lyrique des sciences littéraires après \\ 1945
}

Ludwig Volker

1 So unrecht haben die Puristen unter den Praktikern nicht: die Vorstellung davon, was Lyrik ist oder sein kann, bildet sich wesentlich im Umgang mit Gedichten selbst, und die weitverbreitete Abwehrhaltung gegenüber jeglicher Theorie' ist daher durchaus verständlich, zumindest wenn hinter ihr die Sorge steht, die einfache Wahrnehmung von Gedichten könnte durch gattungspoetische Dogmen oder sekundärästhetische Interessen gestört werden.

Es wäre aber kurzschlüssig gedacht, wollte man den Umgang mit Lyrik überhaupt von Theorie-Reflexion freihalten. Die Lyrik selbst, und dies nicht erst in ihrer neuzeitlichmodernen Entwicklung - hier freilich in gesteigertem Maße -, läßt die Theorie als wichtigen Bestandteil der lyrischen Praxis erkennen, so daß die Grenze zwischen "Theorie“ und „Praxis“ weit weniger scharf zu ziehen ist, als manche gutgemeinte Polemik glauben machen will. Die Behauptung, es könne heutzutage, im Gegensatz zu früheren Zeiten, eine Poetik der Lyrik "gar nicht geben», weil diese «entweder ein Regelwerk wäre, für das sich kaum ein Schriftsteller interessierte, oder eben eine Philosophie, die sich in so große Höhen der Abstraktion begeben müßte, daß die Dichtung selbst darin nicht mehr zu erkennen wäre oder gar verschwände» ${ }^{1}$, vereinfacht die Dinge $\mathrm{zu}$ sehr und verkennt die Bedeutung, welche die Auseinandersetzung mit Fragen der Poetik insbesondere für die Lyrik des 20. Jahrhunderts besitzt. Kaum ein Lyriker (eine Lyrikerin), der (die) sich damit begnügt, einfach Gedichte zu schreiben, und nicht in irgendeiner Form theoretisch über sein (ihr) Tun und das Wesen lyrischer Dichtung reflektiert, - sei es, daß die Gedichte selbst in immanenter poetologischer Reflexion theoretische Aussagen über das Wesen lyrischer Dichtung enthalten oder daß das lyrische Werk durch selbständige Theorie- 
Reflexion in getrennter Textform ergänzt wird, wobei das Spektrum vom Essay und der Rede über die Rezension und das Werkstattgespräch bis zu der in den akademischen Betrieb integrierten Poetologie-Vorlesung reicht ${ }^{2}$.

3 Hinzukommt, daß schon die einfache Wahrnehmung von Gedichten nicht voraussetzungslos und nicht unabhängig von dem erfolgt, was der Leser sich unter einem Gedicht vorstellt und was für Erwartungen er an Lyrik heranträgt. Wie Gedichte gelesen wer- den, welche Bedeutung ihnen im Kontext gesellschaftlicher und kultureller Praxis beigemessen wird, hängt in hohem Maße davon ab, in welcher Weise über Lyrik in der Schule, an der Universität, in den Medien gedacht und gesprochen wird. Mit anderen Worten: der Lyrikbegriff ist keine Sache nur der Lyriker, sondern auch der Lehrer, Professoren, Kritiker. Was eine Epoche, Gesellschaft, Kultur als die besondere Leistung und Bedeutung von Lyrik definiert, ist das Ergebnis einer durch vielfältigen Austausch zwischen den einzelnen Institutionen geprägten Meinungsbildung.

4 Was die deutschen Verhältnisse angeht, so ist das Lyrik- Verständnis hier im Vergleich zu dem anderer Länder von charakteristischen eigenen Akzentsetzungen geprägt. Diese betreffen bestimmte Einstellungen, die weniger das Resultat einer Interpretation empirischer literarischer Fakten als vielmehr der Ausdruck eines Interesses an besonderen grundsätzlichen, allgemeinästhetischen Wert- und Wunschvorstellungen ist.

5 Zwei Optionen sind es vor allem, welche das deutsche Verständnis von Lyrik von seinem relativ späten ${ }^{3}$ Beginn um die Mitte des 18. Jahrhunderts an bestimmen. Die erste Option betrifft die wesentlich auf Herders Einfluß zurückgehende idealtypische Orientierung des Lyrikbegriffs an der Darstellung des „natürlichen“, d.h. unmittelbaren, echten Gefühlsausdrucks: «Ode! sie wird wieder, was sie war! Gefühl ganzer Situation des Lebens! Gespräch Menschlichen Herzens - mit Gott! mit sich! mit der ganzen Natur! $\aleph^{4}$

6 Die zweite Option steht im Zeichen des spekulativen Systemdenkens des deutschen Idealismus und äußert sich zum einen in der Bestimmung der Lyrik als der «subjektiven» Gattung, zum andern in der Einbettung dieses Gattungsverständnisses in ein einheitliches und umfassendes System der Künste und der literarischen Gattungen. Bekanntlich hat Hegel dieser Auffassung den für die Folgezeit verbindlichen Ausdruck gegeben.

7 Beide Optionen haben das deutsche Lyrik-Denken nachhaltig beeinflußt, und man kann sagen, daß die Folgen dieser Prägung von der Literaturwissenschaft erst im 20. Jahrhundert erkannt und analysiert wurden, und zwar sowohl hinsichtlich ihrer Bedeutung für die Geschichte der deutschen Lyrik wie auch ihrer Bedeutung für den Gattungsbegriff als solchen. Erst in dem Maße, wie diese Einseitigkeiten als solche reflektiert wurden, konnten andere lyrik- theoretische Paradigmen ins Bewußtsein treten. Der Prozeß - und Ertrag - dieser Bemühung des akademischen Lyrik-Diskurses nach 1945 um einen revidierten Gattungsbegriff der Lyrik sei im folgenden in einigen kursorischen Strichen angedeutet. 


\section{I.} inzwischen weiß, keinen völligen Neubeginn. Die oft beschworene „Stunde Null“ ist eine Fiktion, die einer genaueren Prüfung nicht standhält ${ }^{5}$. Wie stark die Kontinuität der Tradition über das Jahr 1945 hinaus in Wirklichkeit war, zeigt sich gerade auf dem Gebiet der Gattungspoetik und Lyriktheorie besonders deutlich. führenden Germanisten seiner Zeit, seine Darstellung Die Wissenschaft von der Dichtung mit dem Entwurf einer Systematik der literarischen Gattungen veröffentlicht ${ }^{6}$. Deren Grundlage bildete Goethes gattungspoetische Systemskizze über die «Dichtarten» und «Naturformen der Dichtung» aus den Noten und Abhandlungen zu besserem Verständnis des West-östlichen Divans (1819). Im Schema des «Rades», dessen Speichen die «Urelemente» der drei «Grundgattungen» Epos (= «Bericht»), Lyrik (= «Zustand»), Drama (= «Dialog») darstellten und an dem sich nach Petersens Überzeugung durch «Drehung» das gesamte Spektrum der literarischen Genres mit «allen möglichen Übergängen und Wandlungen» veranschaulichen lie $\aleph^{7}$, offenbarte sich einmal mehr die Abhängigkeit der deutschen Literaturwissenschaft vom Systemdenken des deutschen Idealismus. Die im ersten Nachkriegsjahr 1946 veröffentlichten und mit großer Resonanz aufgenommenen Grundbegriffe der Poetik Emil Staigers blieben dieser Tradition gleichfalls unverkennbar verpflichtet, auch wenn das Bemühen um «fundamentalpoetische» Klärung der «Grundbegriffe» nicht auf die Gattungen (Lyrik, Epik, Dramatik), sondern auf die Ausdruckshaltungen (das Lyrische, Epische, Dramatische) zielte. Im übrigen knüpften Staigers Grundbegriffe, auch dies ein Zeichen wissenschaftsgeschichtlicher Kontinuität, zwar unausgesprochen, aber doch deutlich erkennbar, an Bemühungen seines Lehrers Emil Ermatinger an ${ }^{8}$.

Staigers Traditionsgebundenheit schlägt sich besonders deutlich in seiner Auffassung vom Wesen des «Lyrischen» nieder. Die hierfür zentrale Vorstellung des fehlenden «Abstands»- zwischen Subjekt und Objekt, zwischen Innen und Außen, Sprache und Bewußtsein, Sprachform und Sprachinhalt - liegt ganz auf der Linie traditioneller deutscher Lyrikauffassung, wonach lyrisches Sprechen als ein Sprechen aus der Unmittelbarkeit der Stimmung und des Gefühls heraus anzusehen ist: «Der lyrische Dichter leistet nichts. Er überläßt sich - das will buchstäblich verstanden sein - der Eingebung [...] Sein Dichten ist unwillkürlich.»" Selbst wenn diese Bestimmung sich wie Staiger ausdrücklich bekennt - auf Gedichte der deutschen Romantik als die vermeintlich typischen Erscheinungsformen lyrischer Dichtung bezieht, hätte gerade das von Staiger bevorzugt herangezogene Beispiel der Brentanoschen Lyrik die willkürliche Verengung des Blicks und die Vernachlässigung wesentlich artistischer Momente lehren können. Der Einfluß des lyriktheoretischen Unmittelbarkeitsdenkens wirkte ungebrochen fort. An einer kritischen Überprüfung und Diskussion dieses wichtigen Bestandteils deutscher Lyrik-Theorie, wozu eine bereits 1932 publizierte Untersuchung Gelegenheit geboten hätte ${ }^{10}$, bestand offensichtlich noch kein Bedarf.

11 Die Einseitigkeit der Staigerschen Definition des Lyrischen zeigt sich noch deutlicher vor dem Hintergrund der lyrikgeschichtlichen Entwicklung und wirkt geradezu anachronistisch, versucht man sie auf die für die lyrische Moderne konstitutiven Erscheinungsformen anzuwenden. Deshalb mußten Staigers lyriktheoretische Maßstäbe in dem Maße obsolet werden, in dem sich der deutsche akademische Lyrik- 
Diskurs dieser lyrischen Moderne zuwandte. Und im selben Maße, wie deren historische Relativität durchschaut werden konnte, ergaben sich nicht nur andere lyriktheoretische, sondern auch neue lyrikgeschichtliche Perspektivierungen, wie zum Beispiel die Vorstellung der «Struktureinheit älterer und moderner Lyrik» ${ }^{11}$ und des Wiederanknüpfens an eine ältere, in Deutschland durch den anderen Typus der Lyrik des jungen Goethe und der Romantik nur unterbrochene Lyriktradition ${ }^{12}$.

Die Unvereinbarkeit der Positionen wurde in einem besonders wirkungsmächtigen lyrikgeschichtlichen Ereignis der Nachkriegszeit, Gottfried Benns Marburger Vortrag Probleme der Lyrik von 1951, schlagartig sichtbar, als nämlich Benn gleich zu Beginn die Meinung, Gedichte entstünden aus Stimmungen, als Fehlurteil der öffentlichen Meinung an den Pranger stellte:

... die Öffentlichkeit lebt nämlich vielfach der Meinung: da ist eine Heidelandschaft oder ein Sonnenuntergang, und da steht ein junger Mann oder ein Fräulein, hat eine melancholische Stimmung, und nun entsteht ein Gedicht. Nein, so entsteht kein Gedicht. Ein Gedicht entsteht überhaupt sehr selten - ein Gedicht wird gemacht ${ }^{13}$.

13 Nicht zu überhören war die Anspielung auf Edgar Allan Poes Zentralvorstellung des «Machens» (in The Philosophy of Composition, 1846) und damit die Ausrichtung von Benns Lyrikverständnis an den Mustern der internationalen lyrischen Moderne, die in Staigers Lyrik-Reflexion ausgeklammert waren: Baudelaire, Mallarmé, Valéry, Eliot, Pound.

Die Wirkung Benns als Leitfigur der ersten Nachkriegsjahre - bis dann in den sechziger Jahren das andere Vorbild Brecht ihn ablöste - erstreckte sich auch auf den akademischen Bereich. So steht Hugo Friedrichs 1956 veröffentlichte Studie Die Struktur der modernen Lyrik in deutlicher Abhängigkeit des Bennschen Vortrags, was umsomehr auffällt, als gerade der deutschen Lyrik des zwanzigsten Jahrhunderts in dieser Darstellung des Romanisten eine etwas stiefmütterliche Behandlung zuteil wird.

Hugo Friedrichs Buch, mit zahlreichen Neuauflagen schnell zum wissenschaftlichen Bestseller avanciert, übte seinerseits maß- geblichen Einfluß auf den deutschen akademischen Lyrik-Diskurs aus. Was an Lyrik produziert wurde, wurde gerne unter dem Blickwinkel und mit den Maßstäben wahrgenommen, die Friedrich seiner Darstellung zugrundegelegt hatte: d.h. als Darstellung von Entfremdung, Ich-losigkeit, Leere, mit großer Nähe zu den Zielvorstellungen der „poésie pure“ und des "Hermetismus" und extremer Distanz zur Prosa und zu jeglicher Form gesellschaftlicher Praxis. Ins Normativ-Exemplarische gesteigert konnte dieser LyrikBegriff sogar zum „Paradigma der Moderne“ erhoben werden ${ }^{14}$. Daß es neben der von Friedrich definierten „modernen Lyrik“ noch andere Spielarten der lyrischen Moderne gab, deren Bedeutung mit seinen Kriterien nicht zu erfassen war und die einen anderen lyriktheoretischen und -geschichtlichen Zugriff erforderten, blieb zunächst weitgehend unberücksichtigt ${ }^{15}$.

16 Auf bestimmte problematische Annahmen dieser neuen „deutschen“ Lyriktheorie, die sich in der Nachfolge Hugo Friedrichs und im Umkreis von Hans Robert Jauß und der Gruppe um Poetik und Hermeneutik entwickelte, machte 1971 Paul de Man in dem Kapitel «Lyric and Modernity» seines Buches Blindness and Insight aufmerksam ${ }^{16}$. De Man stellte insbesondere die Stichhaltigkeit eines radikalen Anti-Mimesis-Konzepts für die moderne Lyrik in Frage und wies darauf hin, daß auch bei Mallarmé mimetischreferentielle Momente der Wirklichkeitsdarstellung zu beobachten seien. Die Sprache 
der modernen Lyrik sei keineswegs rein figurativ-allegorisch, sondern ambivalent, d.h. «representational and nonrepresentational at the same time» ${ }^{17}$. Der Gegensatz zwischen „mimetischer" Traditionalität und „allegorischer“, d.h. hermetisch-dunkler und „musikalisch“-selbstreferentieller Modernität sei eine problematische literaturgeschichtliche Konstruktion. In Wahrheit zeige sich an moderner Lyrik nur das allgemeine Paradox der Lyrik, das darin bestehe, daß Lyrik stets von neuem die unerreichbare Antwort auf ihr eigenes Rätsel geben wolle: «The question of modernity reveals the paradoxical nature of a structure that makes lyric poetry into an enigma which never stops asking for the unreachable answer to its own riddle.» ${ }^{18}$

Aus der Distanz des Rückblicks werden die Einseitigkeiten in den lyriktheoretischen Zielvorstellungen moderner Lyrik - ein Kritiker spricht nicht ganz zu Unrecht von "Chimären und Aporien»" ${ }^{19}$ - als Manifestationen eines letztlich utopisch bestimmten Denkens erkennbar: Wie schon das Natur-Denken Herders als Zielbegriff eines kulturkritischen Engagements gesehen werden muß, so sind auch die in der Definition moderner Lyrik zutage tretenden Maßstäbe als extreme Idealvorstellungen zu werten. Bildete bei Herder der auf Wiederherstellung natürlicher Totalität und Ursprünglichkeit gerichtete „Irrationalismus“ der Genieperiode den epochenspezifischen Hintergrund solchen Denkens, so kann als Zeithintergrund für die emphatische Zuspitzung des „modernen“ Lyrik-Begriffs einmal ganz allgemein, wie unter anderem bei Benn deutlich erkennbar, der Nihilismus Nietzschescher Prägung, zum andern der Existenzialismus und das «Zeitalter der Angst» (W.H. Auden) als Epochenstimmung der ersten Nachkriegszeit in Anspruch genommen werden.

Mit dem veränderten Zeitgeist der sechziger und siebziger Jahre schwand auch die Verbindlichkeit des Lyrik-Verständnisses Hugo Friedrichscher Prägung. Parallel zur Politisierung der akademischen Öffentlichkeit begann die Lyrik Bertolt Brechts mit ihren ganz anderen lyriktheoretischen Paradigmen die des Antipoden Benn zu verdrängen. Die Folge für den Lyrik-Begriff war, daß gesellschaftskritische Intentionen und Fragen der moralischen und politischen Verantwortung aus ihrer Randstellung in den Mittelpunkt des lyrischen Zuständigkeitsbereiches rückten. So beleuchtete Michael Hamburger 1969 in einer Art Gegenentwurf zu Hugo Friedrichs Darstellung die Entwicklung der modernen Lyrik seit Baudelaire aus dem Gesichtspunkt der geistigen und kulturellen Rolle, welche dem Lyriker und der Lyrik in der modernen Gesellschaft zufiel $^{20}$. In den autorpoetischen Lyrik-Diskussionen brach man offen mit den Maßstäben der als „modern“ definierten Lyrik ${ }^{21}$ und setzte gegen das „monologischabsolute“, „hermetische“ Gedicht das Gedicht als "Gelegenheitsgedicht" und als "Gebrauchsgegenstand“"22. Und man propagierte eine Lyrik der „neuen Subjektivität“, die im Alltagsleben verwurzelt war und einen kommunikativ-demokratischen FormGestus zu verwirklichen suchte: «Die Republik wird erkennbar, die sich befreit»».

Im akademischen Lyrik-Diskurs öffnete sich der Blick für funktionale, pragmatische, mediale, institutionelle, rezeptionsästhetische sowie ideologiekritische und mentalitätsgeschichtliche Fragestellungen. Galt das wissenschaftliche Interesse an moderner Lyrik bisher vor allem Problemen der Bildlichkeit ${ }^{24}$, so entstanden nun Studien zur politischen Lyrik $^{25}$, zur lyrikgeschichtlichen und gattungspoetischen Bedeutung des „Gelegenheitsgedichts“ ${ }^{\text {“26, }}$, zur Rolle der Lyrik im Geschichtsproze ${ }^{27}$, zur Entwicklung bürgerlicher Subjektivitäts-Vorstellung ${ }^{28}$, zum lyrischen Realismus moderner Großstadt- und Bewußtseinspoesie ${ }^{29}$, zu leserorientierten Aspekten des Lyrik-Begriffs $^{30}$, zur Ideologie und Geschichte des „Erlebnislyrik“-Konzepts ${ }^{31}$ usw. 
20 Mehr oder weniger ausdrücklich gilt für alle diese Arbeiten, was Wulf Segebrecht einleitend über die Entstehung und Begründung seines Untersuchungszieles bemerkt, daß nämlich die Fragestellung «von der heute gegebenen Situation her», d.h. von den «Aporien der gegenwärtigen Lyrik» und aktuellen Diskussion um den Lyrikbegriff entwickelt wurde und daß mit der Rekonstruktion der historischen Bedeutung des Typus' „Gelegenheitsgedicht“ «ein Beitrag zur Poetik der deutschen Lyrik» geleistet werden solle ${ }^{32}$. Ein wesentliches Ergebnis seiner Studie ist denn auch der Nachweis, daß im polemisch bewerteten Verhältnis zwischen „Erlebnislyrik“ und „Gelegenheitsgedicht“ eine grundsätzliche Dichotomie der Lyrik sichtbar wird, deren Bedeutung durch die dichtungs- und lyriktheoretische Verabsolutierung des ErlebnisDenkens Diltheyscher Provenienz verfälscht wurde.

21 Indem sie am Beispiel einzelner lyrikgeschichtlicher und lyriktheoretischer Fragestellungen die Beziehungen zwischen dem Ich, seinem Erleben, dem äußeren Anlaß und der Interpretation dieses Erlebens im (geschriebenen oder gelesenen) Gedicht analysieren, sind diese Arbeiten Dokumente ihrer Zeit, die ein gemeinsamer Fragen- und Interessenzusammenhang verbindet.

22 Demgegenüber ist der akademische Lyrik-Diskurs der achtziger und neunziger Jahre durch einen Pluralismus heterogener, nur selten durch Diskussion verbundener Positionen und Tendenzen gekennzeichnet. Ob sich hieraus ein neues Interessen- und Reflexionsprofil entwickelt, bleibt abzuwarten. Mögliche Kristallisationspunkte könnten sich aus der Reaktion auf die besonderen Lyrik-Affinitäten von <Postmoderne> und <Dekonstruktion> ergeben: liegt es im ersten Fall nahe, die noch zu wenig genutzten Potenzen des Spiel-Begriffs ${ }^{33}$ lyriktheoretisch fruchtbar zu machen, so ist im Hinblick auf das emphatische Sprach- und Poesie-Verständnis, das die einzelnen Positionen der Dekonstruktion kennzeichnet, mit einer verschärften Akzentuierung schon seit langem bestehender und im Umgang mit der Lyrik Celans und Ernst Meisters unvermindert wirksam gebliebener Theorie-Ansätze ${ }^{34} \mathrm{zu}$ rechnen.

\section{II.}

Wie sich bereits mehrfach gezeigt hat, spielen für lyriktheoretisches Denken neben den historischen und erkenntnisleitenden Aspekten immer auch wissenschaftstheoretische und methodologische Fragen eine wichtige Rolle. Im Hinblick auf das zweite kann für den deutschen akademischen Lyrik-Diskurs nach 1945 festgestellt werden, daß das Kriterium der wissenschaftlichen Begründbarkeit der Gattungsdefinition zunehmend Beachtung gefunden hat und daß es im Zuge eines geschärften kritischen Umgangs mit dem Gattungsproblem zumindest in zwei zentralen Punkten zu Klärungen gekommen ist, die als echte Fortschritte gelten können, weil sie den wissenschaftlichen Umgang mit dem, was in Lyrik auf gattungsspezifische Weise zur Darstellung kommt, auf eine klare Grundlage stellen. Diese betreffen zum einen die Präzisierung des besonderen Status, den lyrische „Unmittelbarkeit“ und „Subjektivität“ beanspruchen ; zum andern die genauere Erkenntnis und Definition der formalen Grundbedingungen lyrischer Dichtung.

24 Was das erste angeht, so hat eine terminologische Veränderung, die Einbürgerung des Begriffs des „lyrischen Ich“, die sich im akademischen Lyrik-Diskurs in den siebziger Jahren, nach ersten Anläufen zu Beginn des Jahrhunderts ${ }^{35}$, durchgesetzt hat ${ }^{36}$, wesentlich dazu beigetragen, den für die Lyrik so zentralen Bezug zwischen 
persönlicher Erlebnisunmittelbarkeit und sprachlicher Darstellung mit der nötigen Differenziertheit zu behandeln. Daß die Sprache der Lyrik gerade da, wo sie unmittelbarer Gefühlsausdruck und unverstellte Sprache des Herzens zu sein scheint, ganz stilisiert und künstlich sein kann, und daß der Lyriker keineswegs, wie eine beliebte Vorstellung lautet, einfach «singt, wie der Vogel singt ${ }^{37}$ : dieses für das deutsche Lyrik-Denken so bezeichnende Mißverständnis abzubauen, ist wohl die bedeutsamste Leistung der terminologischen Fixierung des "lyrischen Ich“.

$\mathrm{Da}$ an ihr auch weiterführende prinzipielle Fragen nach dem Wirklichkeitsstatus lyrischer Darstellung reflektiert werden können, zeigte die Auseinandersetzung um Käte Hamburgers Versuch einer Bestimmung des „lyrischen Ich“ als Rede-Subjekt einer «nicht-fiktionalen Aussage» und Abgrenzung der Lyrik von den «fiktionalen» Gattungen Epik und Dramatik ${ }^{38}$. Mit der konsequenten Verankerung des im Gedicht Dargestellten in der Rede- Perspektive des „lyrischen Ich“ wurde der Sinn für die dialektische Spannung zwischen Fiktionalität und Unmittelbarkeit im lyrischen Ausdruck geschärft und die Einsicht gefördert, daß das Gedicht einerseits, im Unterschied etwa zum dramatischen Monolog, unmittel- bare Selbstäußerung - im Hegeischen Sinne: «Ausdruck im Sinne des Sich-Aussprechens» ist und damit Grundform der Dichtung überhaupt, sofern diese als «Sprechen» zu definieren ist ${ }^{39}$; daß es aber andrerseits, als Kunstgebilde und formgewordene Objektivation, immer nur die "poetische Fiktion eines Ausdrucks» ${ }^{40}$ ist.

Ein zweiter wichtiger Fortschritt der akademischen Lyrik- Diskussion in Deutschland nach der Überwindung des spekulativen Gattungsdenkens ${ }^{41}$ betrifft die erneute Hinwendung zu den zentralen Fragen der lyrischen Formgebung. Der akademische Lyrik- Diskurs hatte solche Fragen in dem sicheren Bewußtsein, die doktrinären Formvorstellungen der alten Regelpoetik zu Recht hinter sich gelassen zu haben, lange vernachlässigt. In der lyrischen Praxis hatte sich allerdings immer wieder deren unveränderte prinzipielle Bedeutung gezeigt. Als «neu» und «dringend» bezeichnete Gottfried Benn 1950 in einem seiner späten Gedichte «die Frage nach dem Satzbau»" Zuvor hatte Arno Holz, am Beginn der lyrischen Moderne in Deutschland, seine «Revolution der Lyrik» - «Man revolutioniert eine Kunst nur, indem man ihre Mittel revolutioniert $»^{43}$ - auf das typographische Prinzip der <Mittelachse> gegründet, - das Formprinzip, mit dem die Lyrik nach ihrer Annäherung an den natürlichen Sprachrhythmus der Prosa in den Bereich der Kunst und der Gebundenheit der Form zurückgeholt wurde. Ende der dreißiger Jahre hatte dann Bertolt Brecht, mit ganz anderen Intentionen, aber von derselben künstlerischen Einsicht geleitet, der Frage der Rhythmisierung besondere Aufmerksamkeit geschenkt ${ }^{44}$. Und daß das Bewußtsein für die Bedeutung des Formalen auch nach 1945, trotz aller Liberalität und Lässigkeit im Umgang mit lyrischer Form ${ }^{45}$, keineswegs geschwunden ist, bezeugt u.a. auf höchst amüsante und lehrreiche Weise Hans Magnus Enzensberger in einem 1985 unter fiktivem Verfassernamen herausgegebenen Lehr- und Experimentierbuch mit dem bedeutungsvollen Titel Das Wasserzeichen der Poesie ${ }^{46}$. Alles andere als eine Parodie, wie man vermuten könnte, auf die alte Regelpoetik sucht das Buch in der spielerischen Demonstration von poetischer «Destruktion und Rekonstruktion», wie Harald Hartung es formuliert hat, «einen unverfälschten Begriff vom Handwerk der Poesie» zu vermitteln ${ }^{47}$.

Die Frage nach der Lyrik mit Benn als eine Frage des «Satzbaus» zu bezeichnen, heißt den Blick auf die einfachen prosodischen und rhythmischen Verhältnisse richten und 
damit auf den wohl am meisten in die Augen fallenden Unterschied zwischen Lyrik und Prosa. „Verse machen“ statt „Sätze formulieren“ könnte auf dieser untersten Ebene der Sprachgestaltung das Gattungsprinzip der Lyrik lauten. In der akademischen Lyriktheorie gab es nach 1945 zunächst nur vereinzelte Ansätze einer entsprechenden formtheoretischen Reflexion. Auf Friedrich Georg Jüngers Studien über Rhythmus und Sprache im deutschen Gedicht (1952) folgte eine Abhandlung Beda Allemanns Über das Dichterische (1957), in welcher der Versuch gemacht wurde, das Wesen des Dichterischen über eine Theorie des Rhythmus' zu bestimmen, und zwar im Rückgriff auf Überlegungen August Wilhelm Schlegels zur gattungspoetischen Bedeutung von Prosodie und Rhythmus ${ }^{48}$. Nachdem diese Ansätze lyriktheoretisch nicht weiter verfolgt worden waren, gaben die seit den siebziger Jahren in breiterem Umfang in Deutschland rezipierten Theorie-Ansätze aus dem Bereich des Russischen Formalismus und der Strukturalistischen Poetik neue Anstöße, die Differenz der Lyrik zur Prosa näher zu analysieren und für die Bestimmung des Lyrik-Begriffs zu nutzen. Einen vorläufigen Abschluß haben diese Bemühungen in einer 1989 veröffentlichten gattungspoetischen Grundlegung von Dieter Lamping gefunden ${ }^{49}$.

In kritischer Abgrenzung gegen das traditionelle deutsche Lyrikdenken und unter strikter Beschränkung auf empirisch objektivierbare Merkmale definiert Lamping das lyrische Gedicht als «Einzelrede in Versen» mit den ergänzenden Bestimmungen «monologischer», «absoluter», «strukturell einfacher» Rede. Damit ist ein Gattungsbegriff gewonnen, der es erlaubt, die unterschiedliche Realisierung eines einheitlichen lyrischen Gattungsmusters in den einzelnen prosodischen Systemen und im historischen Wandel ihrer Erscheinungsformen zu beschreiben, wie zum Beispiel in dem für die Moderne besonders charakteristischen Übergang vom traditionellen musikalischen Rhythmisierungsprinzip des „Verses“ zum graphisch-visuellen Gliederungsprinzip der „Zeile“.

29 Mit dieser sehr überzeugenden Gattungsbestimmung scheint die Aporie bisheriger Lyriktheorie überwunden und die Warnung, die René Wellek 1970 als Fazit einer Diskussion der gattungspoetischen Ansätze Staigers und Käte Hamburgers ausgesprochen hat: «Man sollte den Versuch gänzlich aufgeben, die allgemeine Natur der Lyrik oder des Lyrischen bestimmen zu wollen» ${ }^{50}$, gegenstandslos geworden $\mathrm{zu}$ sein.

Daß damit die Divergenzen und Probleme literaturwissenschaftlicher Lyriktheorie bereinigt seien, darf indessen bezweifelt werden. Welche Richtung die weitere Entwicklung des akademischen Lyrik-Diskurses in Deutschland nehmen wird, hängt davon ab, ob man sich mit der erreichten gattungspoetischen Klärung zufrieden geben und darauf verzichten kann - oder will -, jene allgemeinen ästhetischen und philosophischen Fragen weiterhin, wie bisher, mit der Frage nach dem Wesen und der Bedeutung lyrischer Dichtung zu verknüpfen. Das lyriktheoretische Interesse, das sich, wie in Lampings Untersuchung, auf das Phänomen der lyrischen «Rede in Versen» richtet, ist ein anderes als dasjenige, das, wie es in einem jüngst erschienenen Beitrag des akademischen Lyrik- Diskurses der Fall ist, auf das «Denken in Versen», auf die lyrisch gedachte und entworfene «Welt in Versen» zielt ${ }^{51}$. Der Unterschied beider Perspektiven verdeutlicht nicht nur die Spannweite der im akademischen LyrikDiskurs verfolgten Interessen. Er läßt auch das generelle Problem der Literaturwissenschaft sichtbar werden, das darin besteht, einem Gegenstand gerecht 
werden zu müssen, dessen Bedeutung sich der stringenten wissenschaftlichen Analyse und Erkenntnis immer nur in Teilaspekten erschließt.

\section{NOTES}

1. Klaus Schuhmann, Lyrik des 20. Jahrhunderts. Materialien zu einer Poetik. Reinbek 1995. S. 8.

2. Eine interessante neue Form-Variante in diesem Spektrum bietet übrigens der vor kurzem (Basel 1995) erschienene Band Die Schweizer Korrektur, in dem mit dem Mittel des Vierspaltendrucks die gemeinsame dialogisch-kritische Lyrik-Reflexion dreier Autoren dokumentiert ist: Durs Grünbein / Brigitte Oleschinski / Peter Waterhouse, Die Schweizer Korrektur. Basel 1995.

3. Zur geschichtlichen Entwicklung der deutschen Lyriktheorie vgl.: Ludwig Völker (Hg.), Lyriktheorie. Texte vom Barock bis zur Gegenwart. Stuttgart 1990.

4. Johann Gottfried Herder, Auszug aus einem Briefwechsel über Ossian und die Lieder alter Völker (1773). In: Herder / Goethe / Frisi / Moser, Von deutscher Art und Kunst. Hg. von Hans Dietrich Irmscher. Stuttgart 1988, S. 60.

5. Vgl.: Lothar Köhn, Vom Nullpunkt zum Posthistoire. Zu Konstruktionselementen einer Geschichte der deutschen Literatur 1945-1990. In: 1945-1995. Fünfzig Jahre deutschsprachiger Literatur in Aspekten. Hg. von Gerhard P. Knapp und Gerd Labroisse. Amsterdam 1995, S. 13-55.

6. Lyriktheorie S. $165 \mathrm{f}$.

7. Julius Petersen, Die Wissenschaft von der Dichtung. 2. Aufl. Berlin 1944, S. 126.

8. Emil Ermatinger: Das dichterische Kunstwerk. Grundbegriffe der Urteilsbildung in der Literaturgeschichte. Leipzig 1921.

9. Emil Staiger: Grundbegriffe der Poetik. 5. Aufl. München 1983, S. 19.

10. Ernst Voege: Mittelbarkeit und Unmittelbarkeit in der Lyrik. Untersuchungen an lyrischen Gedichten des Altertums und der Neuzeit im Hinblick auf die herrschende deutsche LyrikTheorie. München 1932. Neudruck Darmstadt 1968.

11. Vgl.: Hans Robert Jauß, Zur Frage der «Struktureinheit» älterer und moderner Lyrik. In: Germanisch-Romanische Monatsschrift N.F. 10 (1960), S. 231-266. Wiederabgedruckt in: Reinhold Grimm (Hg.), Zur Lyrik-Diskussion. Darmstadt 1966, S. 314 ff.

12. Vgl.: Karl Otto Conrady, Moderne Lyrik und die Tradition. In: Germanisch-Romanische Monatsschrift N.F. 10 (1960), S. 287-304. Wiederabgedruckt in: Reinhold Grimm (Hg.), Zur LyrikDiskussion. Darmstadt 1966, S. 411 ff.

13. Lyriktheorie S. 359.

14. Wolfgang Iser (Hg.), Immanente Ästhetik - Ästhetische Reflexion. Lyrik als Paradigma der Moderne. München 1966.

15. Vgl. hierzu: Ludwig Völker, Lyrik als «Paradigma der Moderne»? In: Zs.f. Germanistik N.F. 3 (1993), S. 487-500.

16. Paul de Man, Lyric and Modernity, in: P.d.M., Blindness and Insight. Essays in the Rhetoric of Contemporary Criticism. New York 1971, S. 166-186.

17. de Man, a.a.O. S. 185.

18. de Man, a.a.O. S. 186.

19. Hans-Martin Gauger, Gibt es eine Sprache der Moderne? In: Heinrich Meier (Hg.), Zur Diagnose der Moderne. München 1990, S. 173-210. 
20. Michael Hamburger: The Truth of Poetry (1969) ; deutsche Ausgaben: Die Dialektik der modernen Lyrik. Von Baudelaire bis zur konkreten Poesie. München 1972 ; Wahrheit und Poesie. Spannungen in der modernen Lyrik von Baudelaire bis zur Gegenwart. Berlin 1985.

21. Vgl. u.a.: Jürgen Theobaldy / Gustav Zürcher, Veränderung der Lyrik. Über westdeutsche Gedichte seit 1965. München 1976.

22. Vgl.: Hans Magnus Enzensberger, Scherenschleifer und Poeten, in: Hans Bender (Hg.), Mein Gedicht ist mein Messer. Lyriker zu ihren Gedichten. München 1961, S. 144-148; Günter Grass, Das Gelegenheitsgedicht, in: Akzente 8 (1961), S. 8-11.

23. Walter Höllerer, Thesen zum langen Gedicht (1965) ; zit. nach: Lyriktheorie S. 403.

24. Vgl. u.a.: Clemens Heselhaus, Deutsche Lyrik der Moderne von Nietzsche bis Yvan Göll. Die Rückkehr zur Bildlichkeit der Sprache. Düsseldorf 1961 ; Walther Killy, Wandlungen des lyrischen Bildes. Göttingen 1956 ; Edgar Marsch, Die lyrische Chiffre. Ein Beitrag zur Poetik des modernen Gedichts. In: Sprachkunst 1 (1970), S. 207-240.

25. Vgl. u.a.: Albrecht Schöne, Über politische Lyrik im 20. Jahrhundert. Göttingen 1965 ; Ingrid Girschner-Woldt, Theorie der modernen politischen Lyrik. Berlin 1971.

26. Wulf Segebrecht, Das Gelegenheitsgedicht. Ein Beitrag zur Geschichte und Poetik der deutschen Lyrik. Stuttgart 1977.

27. Walter Hinck, Von Heine zu Brecht. Lyrik im Geschichtsprozeß. Frankfurt a.M. 1978.

28. Hiltrud Gnüg, Entstehung und Krise lyrischer Subjektivität. Vom klassischen lyrischen Ich zur modernen Erfahrungswirklichkeit. Stuttgart 1983.

29. Gottfried Willems, Großstadt- und Bewußtseinspoesie. Über Realismus in der modernen Lyrik, insbesondere im lyrischen Spätwerk Gottfried Benns und in der deutschen Lyrik seit 1965. Tübingen 1981.

30. Elke Austermühl, Poetische Sprache und lyrisches Verstehen. Studien zum Begriff der Lyrik. Heidelberg 1981.

31. Michael Feldt, Lyrik als Erlebnislyrik. Zur Geschichte eines Literatur- und Mentalitätstypus zwischen 1600 und 1900. Heidelberg 1990.

32. Segebrecht, a.a.O. S. 14.

33. Vgl. Alfred Liede, Dichtung als Spiel. Studien zur Unsinnspoesie an den Grenzen der Sprache. Berlin 1963. 2., erw. Aufl. Berlin 1992. - Obgleich das von Liede untersuchte Textmaterial nahezu ausschließlich der lyrischen Gattung angehört, wird die lyriktheoretische Bedeutung des SpielBegriffs nicht reflektiert.

34. Vgl. u.a.: Helmut Arntzen, Der Literaturbegriff. Geschichte, Komplementärbegriffe, Intention. Eine Einführung. Münster 1984 ; Johannes Anderegg, Sprache und Verwandlung. Göttingen 1985.

35. Vgl. Lyriktheorie S. $290 \mathrm{ff}$. und S. $297 \mathrm{ff}$.

36. Vgl. u.a.: Karl Pestalozzi, Die Entstehung des lyrischen Ich. Studien zum Motiv der Erhebung in der Lyrik. Berlin 1970 ; Kaspar H. Spinner, Zur Struktur des lyrischen Ich. Frankfurt a.M. 1975 ; Wolfgang G. Müller, Das lyrische Ich. Erscheinungsformen gattungseigentümlicher AutorSubjektivität in der englischen Lyrik. Heidelberg 1979.

37. Nach Goethes Ballade Der Sänger ; vgl. Lyriktheorie S. 251.

38. Käte Hamburger, Die Logik der Dichtung (1957); vgl. Lyriktheorie S. 405 ff.

39. Arntzen, a.a.O. S. 100 und S. 103.

40. Arntzen, a.a.O. S. 105.

41. Vgl. hierzu: Gottfried Willems, Das Konzept der literarischen Gattung. Untersuchungen zur klassischen deutschen Gattungstheorie, insbesondere zur Ästhetik F. Th. Vischers, Tübingen 1981.

42. Satzbau, in: Gottfried Benn, Sämtliche Werke. Stuttgart 1986 ff. Bd. I, S. 238.

43. Lyriktheorie S. 270.

44. Vgl. u.a. Brechts Aufsatz Über reimlose Lyrik mit unregelmäßigen Rhythmen (1938). 
45. Kritisch hierzu: Peter Wapnewski, Gedichte sind genaue Form (1977) ; vgl. Lyriktheorie S. 424 ff.

46. Das Wasserzeichen der Poesie oder Die Kunst und das Vergnügen, Gedichte zu lesen. In hundertvierundsechzig Spielarten vorgestellt von Andreas Thalmayr. Nördlingen 1985.

47. Harald Hartung: Der Trichter ins Freie. Andreas Thalmayrs «Wasserzeichen der Poesie». In: Merkur 40 (1986), S. 58-61.

48. August Wilhelm Schlegel, Briefe über Poesie, Silbenmaß und Sprache (1795/96); vgl. Lyriktheorie S. $131 \mathrm{ff}$.

49. Dieter Lamping, Das lyrische Gedicht. Definitionen zu Theorie und Geschichte der Gattung. Göttingen 1989, 2. Aufl. 1993.

50. René Wellek: Gattungstheorie, das Lyrische und Erlebnis. In: R.W., Grenzziehungen. Beiträge zur Literaturkritik. Stuttgart 1972, S. 106-124 (Zitat: S. 124).

51. Ulrich Schödlbauer, Entwurf der Lyrik. Berlin 1994, S. 25.

\section{RÉSUMÉS}

Wissenschaft, Unterricht, Medien beeinflussen in nicht unerheblichem Ausmaß die herrschenden Vorstellungen über das Wesen lyrischer Dichtung. Der wissenschaftliche Lyrik-Diskurs in Deutschland nach 1945 ist durch starke Umbrüche und lyriktheoretische Neuorientierungen bestimmt. Die traditionelle Macht gattungspoetischen Systemdenkens wurde gebrochen, typisch 'deutsche' Einstellungen zur Lyrik als Darstellung eines unmittelbaren Gefühlsausdrucks oder subjektiver Weltsicht wurden terminologisch eingegrenzt und historisch relativiert. Die poetologischen Maßstäbe der lyrischen Moderne, die nach dem Ende des NS-Regimes in Deutschland neu zu rezipieren waren, wurden in den sechziger und siebziger Jahren von pragmatischen und gesellschaftskritischen Interessen verdrängt. Ende der achtziger Jahre wurde in verstärkter methodologischer Reflexion und auf der Basis formalistisch-strukturalistischer Ansätze eine neue Definition des lyrischen Gedichts gewonnen, die dazu beitragen kann, bisherige Aporien der deutschen Lyriktheorie zu überwinden.

La science, l'enseignement et les médias ne sont pas sans influencer considérablement les représentations dominantes sur la nature que nous offre la poésie lyrique. Le discours scientifique sur le lyrisme dans l'Allemagne d'après 1945 est déterminé par de profondes mutations et par les nouvelles orientations prises par les théories sur le lyrisme. On battit en brèche le pouvoir traditionnellement exercé par une pensée de type systémique, axée sur une poétique des genres, et une conception typiquement «allemande» du lyrisme qui faisait de celuici la représentation de l'expression du sentiment ou d'une vision subjective du monde se vit imposer des limites terminologiques, tandis que la référence au contexte historique permettait de la relativiser. Les critères poétologiques de la modernité en poésie, que la fin du nazisme avait obligé à considérer d'un œil nouveau, furent, dans les années soixante et soixante-dix, l'objet d'un refoulement dicté par des intérêts pragmatiques et tournés vers la critique sociale. A la fin des années quatre-vingt, le renforcement de la réflexion méthodologique amena à redéfinir ce qu'est le poème lyrique sur la base de présupposés issus du formalisme structuraliste, définition nouvelle qui peut contribuer à dépasser les apories auxquelles aboutissait jusqu'ici la théorie lyrique allemande. 
AUTEUR

LUDWIG VOLKER

Universität Münster 